RESEARCH NOTES

\title{
CODIMENSION 2 FIBRATORS THAT ARE CLOSED UNDER FINITE PRODUCT
}

\author{
YOUNG HO IM \\ Department of Mathematics \\ Pusan National University \\ Pusan 609-735, KOREA \\ MEE KWANG KANG \\ Department of Mathematics \\ Dongeui University \\ Pusan 614-714, KOREA \\ KI MUN WOO \\ Department of Mathematics \\ Pusan National University \\ Pusan 609-735, KOREA
}

(Received March 14, 1996 and in revised form June 5, 1996)

\begin{abstract}
In this paper, we show that if $N^{m}$ is a closed manifold with hyperhopfian fundamental group, $\pi_{2}(N)=0$ for $1<i \leq n$ and $S^{n}$ is a simply connected manifold, then $N^{m} \times S^{n}$ satisfies the property that all proper, surjective maps from an orientable $(n+2)$-manifold $M$ to a 2-manifold $B$ for which each $p^{-1}(b)$ is homotopy equivalent to $N^{m} \times S^{n}$ necessarily are approximate fibrations.
\end{abstract}

KEY WORDS AND PHRASES: Approximate fibration, Hopfian group, hyperhopfian group, Hopfian manifold.

1991 AMS SUBJECT CLASSIFICATION CODES: 57N15; 55R65.

\section{INTRODUCTION}

In the study of proper maps between manifolds, the concepts of approximate fibrations play very important roles because it has nice and useful properties just as Hurewicz fibrations. As a generalization of Hurewicz fibrations and cell-like maps, the concept of an approximate fibration is initially introduced by Coram and Duvall [1].

A proper map $p: M \rightarrow B$ between locally compact ANR's is called an approximate fibration if it has the following approximate homotopy lifting property for all spaces: given an open cover $\epsilon$ of $B$, an arbitrary space $X$, and two maps $g: X \rightarrow M$ and $X \times I \rightarrow b$ such that $p \circ g=F_{0}$, there exists a map $G: X \times I \rightarrow M$ such that $G_{0}=g$ and $p \circ G$ is $\epsilon$-close to $F$.

If a proper map $p: M \rightarrow B$ is an approximate fibration, then the point inverses are homotopy equivalent to each other. Naturally, the question arises as to under what conditions the converse of this fact holds. Many people have been interested in a setting which forces any proper map defined on an arbitrary manifold of a specified dimension to be an approximate fibration merely due to the fact that all point preimages are copies of some closed manifold $N$. In this paper, we mainly concentrate on such a closed $n$-manifold when the dimension of $M$ is particularly $n+2$.

We assume all spaces are locally compact, metrizable ANR's, and all manifolds are finite dimensional, orientable, connected and boundaryless. A manifold $M$ is said to be closed if $M$ is compact, connected and boundaryless. A closed $n$-manifold $N$ is called a codimension 2 fibrator if, whenever $p: M \rightarrow B$ is a proper map from an arbitrary $(n+2)$-manifold $M$ to a 2-manifold $B$ such that each point preimage : homotopy equivalent to $N, p: M \rightarrow B$ is an approximate fibration.

The degree of a map $R: N \rightarrow N$, where $N$ is a closed manifold, is the nonnegative integer $d$ such that the induced endomorphism of $H_{n}(N ; Z) \simeq Z$ amounts to multiplication by $d$, up to sign Note that 
a degree one map $R: N \rightarrow N$ induces homology isomorphisms $R_{*}: H_{2}(N) \rightarrow H_{i}(N)$ for all integers $i \geq 0$. The continuity set $C$ of $p: M \rightarrow B$ consists of those points $c \in B$ such that, under any retraction $R: p^{-1} U \rightarrow p^{-1} c$ defined over a neighborhood $U \subset B$ of $c, c$ has another neighborhood $V_{c} \subset U$ such that $R \mid p^{-1} b: p^{-1} b \rightarrow p^{-1} c$ is a degree one map for all $b \in V_{c}$. The continuity set $C$ is the maximal open subset of $B$ over which the nth cohomology sheaf of the map $p$ is locally constant. In [2], several characterizations for an approximate fibration were described. As a matter of fact, the essential point whether or not a proper map is an approximate fibration depends on the fact that any retraction $R: p^{-1} U \rightarrow p^{-1} b$ restricts to homotopy equivalences $p^{-1} c \rightarrow p^{-1} b$ for all $c$ sufficiently close to $b$ [2] The following terms efficiently aid to convert a homology equivalence into a homotopy equivalence.

A closed manifold $N$ is called Hopfian if every degree one map $N \rightarrow N$ which induces a $\pi_{1^{-}}$ automorphism is a homotopy equivalence. A group $H$ is Hopfian if every epimorphism $\Psi: H \rightarrow H$ is necessarily an isomorphism, while a finitely presented group $H$ is hyperhopfian if every endomorphisn $\Psi: H \rightarrow H$ with $\Psi(H)$ normal and $H / \Psi(H)$ cyclic is an automorphism. A hyperhopfian group implies a Hopfian one but the converse does not hold.

Daverman ([3],[4]) has shown that each of a simply connected closed manifold and an aspherical closed manifold with hyperhopfian fundamental group is a codimension 2 fibrator. The problem whether the class of codimension 2 fibrators is closed under finite product is not yet settled. A particular class of all closed surfaces with negative Euler characteristics is closed, which is claimed by $\operatorname{Im}([5])$. Also we extended this result to the extent that any product of an $n$-sphere $S^{n}(n>1)$ and a finite product or closed surfaces of genus at least 2 is a codimension 2 fibrator ([6]).

The purpose of this paper is to extend the above results so that any finite product of a simply connected closed manifold $S^{n}$ and a closed manifold $N^{m}$ with hyperhopfian fundamental group and $\pi_{\imath}(N)=0$ for $1<i \leq n$ is a codimension 2 fibrator.

\section{PRELIMINARIES}

The investigation about codimension 2 fibrators compared with other codimensions is easier to approach due to the following result.

LEMMA 2.1 [6]. If $G$ is an upper semicontinuous decomposition of an $(n+2)$-manifold $M$ into closed $n$-manifolds, then the decomposition space $B(=M / G)$ is a 2-manifold and $D=B \backslash C$ is locally finite in $B$, where $C$ represents the continuity set of the decomposition map $p: M \rightarrow B$

Because of the local finiteness of $D$, we can localize the problem to that of an open disk $B$, proved that $p$ is an approximate fibration over the continuity set $C$, so that $p: M \rightarrow B$ is an approximate fibration over $B-b$ for some $b \in B$. In such a case, the homotopy exact sequence for $p$ over $B \backslash b$ can be reduced to a short exact sequence as follows:

$$
0 \simeq \pi_{2}(B-b) \rightarrow \pi_{1}\left(p^{-1}(c)\right) \rightarrow \pi_{1}\left(M-p^{-1}(b)\right) \rightarrow \pi_{1}(B-b) \simeq Z \rightarrow 0,
$$

where $c$ is any point of $B \backslash b$. Hence, if each preimage of $p$ has the same homotopy type as $N$, the hyperhopfian property of $\pi_{1}(N)$ makes the continuity set $C$ the whole set $B$. And so, the hyperhopfian property is very valuable on discussing codimension 2 fibrators.

We state the established results about codimension 2 fibrators.

THEOREM 2.2 [4]. All closed, Hopfian manifolds with hyperhopfian fundamental group is a codimension 2 fibrator.

THEOREM 2.3 [3]. Every simply connected closed manifold is a codimension 2 fibrator.

The idea of the proof is to show that the continuity set of the decomposition map $p: M^{n+2} \rightarrow M / G$ is the entire set $M / G$.

A closed surface with negative Euler characteristic has a hyperhopfian fundamental group [4] Therefore, from Theorem 2.2 and 2.3, the following corollary follows 
COROLLARY 2.4. Every closed surface $F^{2}$ with nonzero Euler characteristic is a codimension 2 fibrator.

THEOREM 2.5 [5]. Any finite product $N=F_{1} \times F_{2} \times \cdots \times F_{m}$ of closed surfaces $F_{i}^{2}(i=1, \cdots, m)$ of genus at least 2 is a codimension 2 fibrator.

Since an $n$-sphere $S^{n}$ is a simply connected closed manifold, each of the fundamental group and the first homology group of $S^{n} \times F_{1} \times \cdots \times F_{m}$ is isomorphic to each of those of $F_{1} \times \cdots \times F_{m}$. Taking advantage of the method in the proof of Theorem 2.5 and the fact that $S^{n} \times F_{1} \times \cdots \times F_{m}$ is a Hopfian manifold, we can obtain the next consequence.

THEOREM 2.6. A finite product $N=S^{n} \times F_{1} \times \cdots \times F_{m}$ of $n$-sphere $S^{n}(n>1)$ and closed orientable surfaces $F_{i}^{2}(i=1, \cdots, m)$ with negative Euler characteristics is a codimension 2 fibrator.

\section{MAIN RESULTS}

PROPOSITION 3.1. Let $S$ be a simply connected closed $n$-manifold and $N$ be a closed $m$ manifold with $\pi_{2}(N)=0$ for $1<i \leq n$. If $R: S \times N \rightarrow S \times N$ is a degree one map, then so is $p r \circ R \circ i: S \rightarrow S$, where $p r: S \times N \rightarrow S$ is the first projection and $i: S \rightarrow S \times N$ is an inclusion map.

PROOF. Assume that $R: S \times N \rightarrow S \times N$ is a degree one map. By taking a universal covering space $(\tilde{N}, \theta)$ of $N,(S \times \tilde{N}, i d \times \theta)$ is a universal covering space of $S \times N$.

Let $\tilde{i}: S \rightarrow S \times \tilde{N}$ be a continuous map for which $(i d \times \theta) \circ \tilde{i}=i$ and $\tilde{R}: S \times \tilde{N} \rightarrow S \times \tilde{N}$ be a continuous map such that $R \circ(i d \times \theta)=(i d \times \theta) \circ \tilde{R}$ by the lifting property. Consider the following commutative diagram

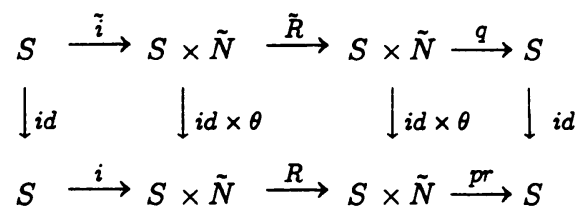

where $q$ is the projection from $S \times \tilde{N}$ onto $S$. Because of $\pi_{2}(N)=0$ for $1<i \leq n$, we obtain that $\pi_{i}(\tilde{N})=0$ for $1 \leq i \leq n$, and then $H_{i}(\tilde{N})=0$ for $1 \leq i \leq n$. According to the Künneth Theorem, $H_{n}(S \times \tilde{N}) \simeq H_{n}(S)$ and $H_{n}(S \times N)$ is isomorphic to the direct sum of $\oplus_{\imath=0}^{n} H_{n-\imath}(S) \otimes H_{\imath}(N)$ and $\oplus_{i=0}^{n-1} H_{n-\imath-1}(S) * H_{i}(N)$. Then it easily checked that $i_{*} \circ q_{*}=(i d \times \theta)$. as homomorphisms from $H_{n}(S \times N)$ to itself, and by the diagram chasing, $R_{*}\left(H_{n}(S)\right) \subset H_{n}(S)$ holds when we restrict $R_{*}$ to $H_{n}(S) \subset H_{n}(S \times N)$.

Rewrite $H_{n}(S \times N)$ in a form \{torsion-free $\oplus$ \{torsion\}. Since $R_{*}$ is an isomorphism, the restriction of $R_{*}$ to \{torsion-free\} of $H_{n}(S \times N)$ is an isomorphism and induces an invertible $k \times k$ matrix of the following form

$$
K=\left(\begin{array}{cccc}
K_{11} & K_{12} & \ldots & K_{1 k} \\
K_{21} & K_{22} & \ldots & K_{2 k} \\
\ldots \ldots \ldots \ldots \ldots \ldots \ldots \ldots . \\
\ldots \ldots \ldots \ldots \ldots \ldots \ldots \ldots \ldots \ldots \\
\hdashline K_{k 1} & K_{k 2} & \ldots & K_{k k}
\end{array}\right)
$$

Here $K_{11}$ is the matrix corresponding to the map $R_{*} \mid H_{n}(S): H_{n}(S) \rightarrow H_{n}(S)$ and $K_{i j}$ is the matrix induced by the homomorphism from the $\mathrm{i}$-th direct summand to the $\mathrm{j}$-th direct summand of torsion-free part of $H_{n}(S \times N)$.

Since $R_{*}\left(H_{n}(S)\right) \subset H_{n}(S)$, the restriction $R_{*} \mid\left\{\right.$ torsion-free\} of $R_{*}$ doesn't send first factor $H_{n}(S)$ to any direct summand except itself and thus $K_{1 j}$ is zero for each $j=2, \cdots, k$. Hence, the isomorphism $R_{*} \mid$ \{torsion-free\} induces $\operatorname{det} K= \pm 1$ and $\operatorname{det} K_{11}= \pm 1$, so that $p r \circ R \circ i$ is a degree one map.

COROLLARY 3.2. Let $S$ be a simply connected closed $n$-manifold and $N$ be a closed aspherical $m$-manifold. If $R: S \times N \rightarrow S \times N$ is a degree one map, then so is $p r \circ R \circ i: S \rightarrow S$, where $p r: S \times N \rightarrow S$ is the first projection and $i: S \rightarrow S \times N$ is an inclusion map. 
COROLLARY 3.3. Let $S$ be a simply connected closed $n$-manifold and $N$ be a finite product of closed orientable surfaces with nonpositive Euler characteristics. Then for every degree one map

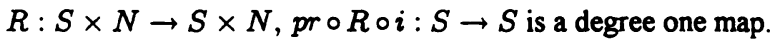

PROOF. Every closed orientable surface except 2-sphere is aspherical and their finite product is a closed aspherical manifold.

REMARK. In the above corollary, a degree one map $R: S_{1} \times S_{2} \rightarrow S_{1} \times S_{2}$ on a product of closed simply connected manifolds $S_{1}$ and $S_{2}$ cannot guarantee that the degree of the restriction $R \mid S_{1}$ is one, so that the condition nonpositive Euler characteristic cannot be omitted.

The following theorem is the main result in this section.

THEOREM 3.4. Let $S$ be a closed simply connected manifold, and $N$ be a closed manifold with hyperhopfian fundamental group and $\pi_{2}(N)=0$ for $1<i \leq n$. Then $S \times N$ is a codimension 2 fibrator.

PROOF. Since the fundamental group of $S \times N$ is isomorphic to the hyperhopfian group $\pi_{1}(N)$, it suffices to show that $S \times N$ is a Hopfian manifold by means of Theorem 2.2. Assume that $R: S \times N \rightarrow S \times N$ is a degree one map. Since a degree one map between compact orientable manifolds of the same dimension induces a $\pi_{1}$-epimorphism ([8]), $R_{\sharp}: \pi_{1}(S \times N) \rightarrow \pi_{1}(S \times N)$ is an epimorphism and actually it is a $\pi_{1}$-isomorphism by the Hopfian property of $\pi_{1}(N)$.

To claim that $R$ is a homotopy equivalence, let us consider $R_{\|}: \pi_{\mathfrak{i}}(S \times N) \rightarrow \pi_{\mathfrak{i}}(S \times N)$ for $i \geq 2$. By Proposition 3.1, the degree of $p r \circ R \circ i: S \rightarrow S$ is one and so $p r_{*} \circ R_{*} \circ i_{*}: H_{2}(S) \rightarrow H_{2}(S)$ is an isomorphism for each $i \geq 1$. Since $S$ is simply connected, we can apply the Whitehead theorem and obtain that $(p r \circ R \circ i)_{\#}: \pi_{i}(S) \rightarrow \pi_{i}(S)$ is a $\pi_{i}$-isomorphism for $i \geq 1$. Implying the fact that $\pi_{i}(S \times N) \simeq \pi_{i}(S) \times \pi_{i}(N) \simeq \pi_{i}(S)$ for each $i \geq n, R_{\sharp}: \pi_{i}(S \times N) \rightarrow \pi(S \times N)$ is an isomorphism for each $i \geq n$. Since $R$ has the property $R \simeq$, we obtain that $R$ is a homotopy equivalence. Therefore, $S \times N$ is a Hopfian manifold.

COROLLARY 3.5. Let $S$ be a closed simply connected manifold, and $N$ be a closed aspherical manifold with hyperhopfian fundamental group. Then $S \times N$ is a codimension 2 fibrator.

COROLLARY 3.6. Let $S$ be a closed simply connected manifold, and $F$ be a closed surface with nonzero Euler characteristic. Then $S \times F$ is a codimension 2 fibrator.

PROOF. If the Euler characteristic of $F$ is negative, its fundamental group is hyperhopfian [4]. Otherwise, $F$ is homeomorphic to 2 -sphere and then $S \times F$ is simply connected. Hence, it is a codimension 2 fibrator.

COROLLARY 3.7. Let $S$ be a closed simply connected manifold, and $N$ be a finite product of closed surfaces with nonzero Euler characteristic. Then $S \times N$ is a codimension 2 fibrator.

PROOF. If a closed surface $F$ has a positive Euler characteristic, then $F$ is a 2-sphere. Therefore, we can rewrite $S \times N$ as $S^{\prime} \times N^{\prime}$, where $S^{\prime}$ is simply connected and $N^{\prime}$ is a product of closed surfaces with negative Euler characteristic. Since $N^{\prime}$ is a closed aspherical manifold with hyperhopfian fundamental group [4], $S \times N$ is a codimension 2 fibrator.

ACKNOWLEDGEMENT. The present studies were supported by the Basic Science Research Institute Program, Ministry of Education, 1996, Project No. BSRI-96-1433.

\section{REFERENCES}

[1] CORAM, D. and DUVALL, P., Approximate fibrations, Rocky Mountain J. Math. 7 (1977), 275-288.

[2] CORAM, D. and DUVALL, P., Approximate fibrations and a movability condition for maps, Pacific $J$. Math. 72 (1977), 41-56.

[3] DAVERMAN, R.J., Submanifold decompositions that induce approximate fibrations, Top. Appl. 33 (1989), 173-184.

[4] DAVERMAN, RJ., Hyperhopfian groups and approximate fibrations, Compositio Math. 86 (1993), 159-176.

[5] IM, Y.H., Decompositions into codimension two submanifolds that induce approximate fibrations, Topology Appl. 56 (1994), 1-11.

[6] IM, Y.H., KANG, M.K. and WOO, K.M., Product spaces that induce approximate fibrations, J. Korean Math. Soc. 33 (1996), 145-154.

[7] DAVERMAN, RJ. and WALSH, J.J., Decompositions into codimension two manifolds, Trans. Amer. Math. Soc. 288 (1985), 273-291.

[8] HEMPEL, J., 3-Manifolds, Princeton University Press, Princeton, NJ, 1976. 


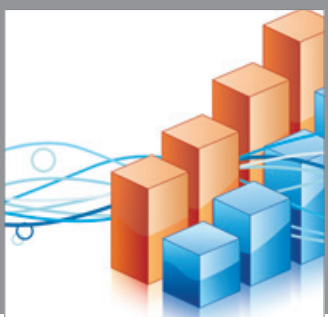

Advances in

Operations Research

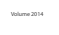

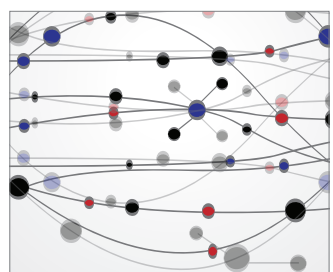

\section{The Scientific} World Journal
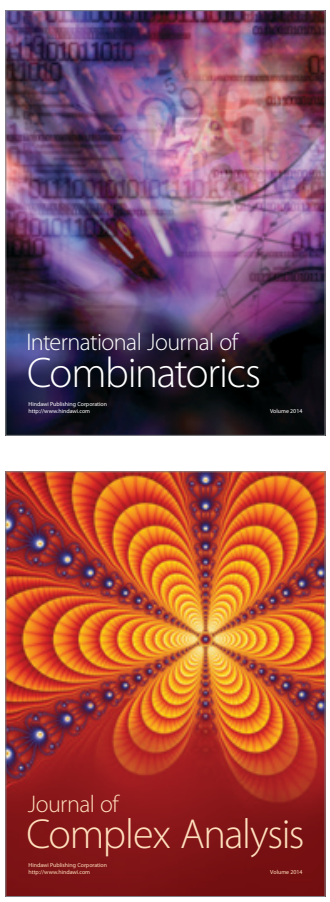

International Journal of

Mathematics and

Mathematical

Sciences
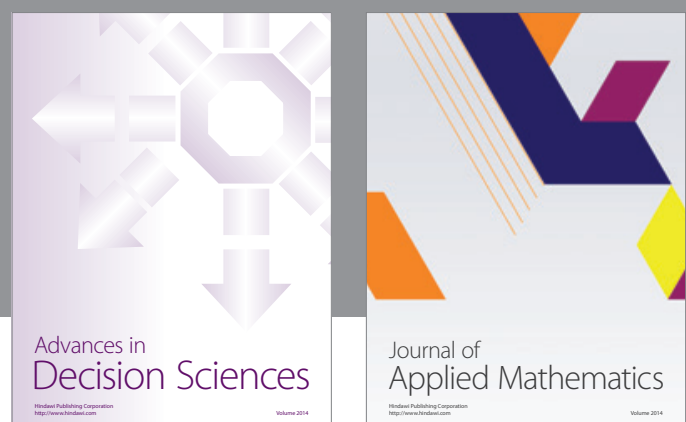

Journal of

Applied Mathematics
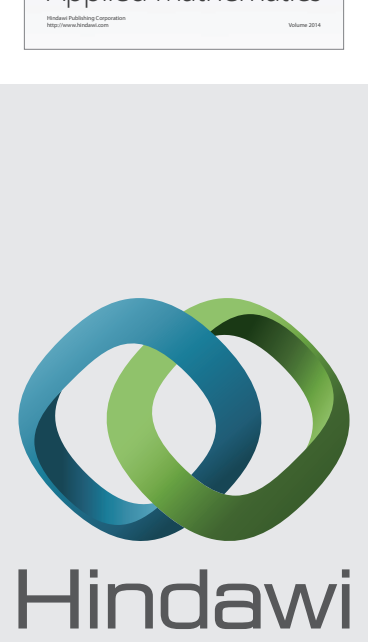

Submit your manuscripts at http://www.hindawi.com
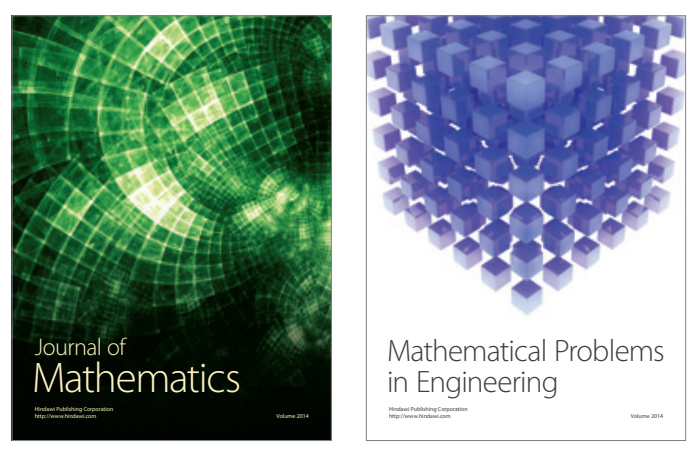

Mathematical Problems in Engineering
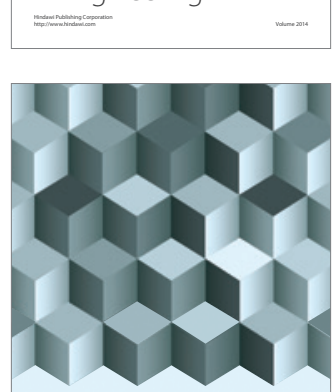

Journal of

Function Spaces
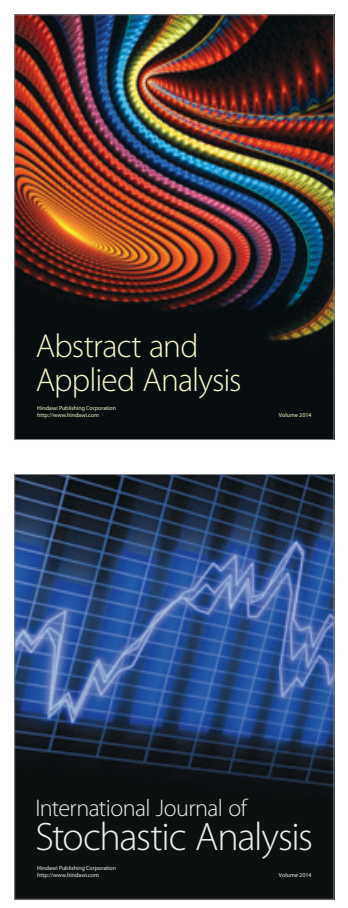

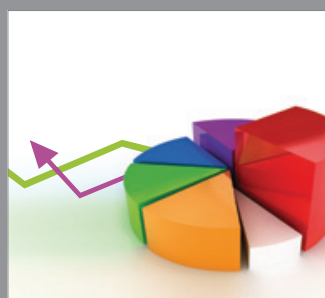

ournal of

Probability and Statistics

Promensencen
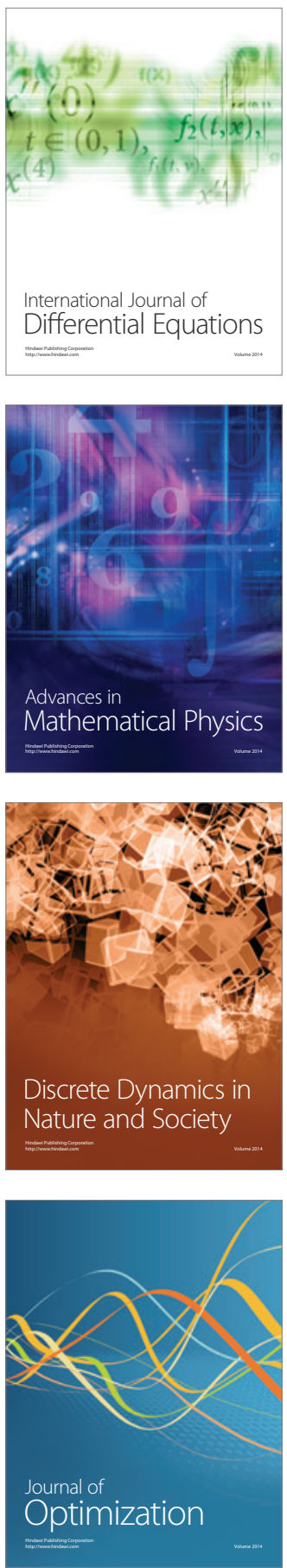